\title{
Protective effect of pterostilbene on sepsis-induced acute lung injury in a rat model via the JAK2/STAT3 pathway
}

\author{
Hua Xue ${ }^{1}$, Manxiang $\mathrm{Li}^{2} \wedge$ \\ ${ }^{1}$ Xi' an Jiaotong University Health Science Center, Xi'an, China; ${ }^{2}$ Department of Respiratory and Critical Care Medicine, The First Affiliated \\ Hospital of Xi'an Jiaotong University, Xi'an, China \\ Contributions: (I) Conception and design: All authors; (II) Administrative support: M Li; (III) Provision of study materials or patients: H Xue; (IV) \\ Collection and assembly of data: H Xue; (V) Data analysis and interpretation: All authors; (VI) Manuscript writing: All authors; (VII) Final approval \\ of manuscript: All authors. \\ Correspondence to: Manxiang Li. 277 West Yanta Road, Xi'an 710061, China. Email: limanxiangxa@sina.com.
}

\begin{abstract}
Background: Bacterial infection is one of the most common causes of sepsis, with acute lung injury (ALI) being a related complication. Pterostilbene (PTS) is extracted from blueberries, peanuts, and grapes, and has numerous pharmacologic activities. The aim of the present study was to explore the underlying role of PTS protects against sepsis-mediated ALI.

Methods: We established a sepsis model induced by cecal ligation and puncture (CLP) in rats. The rats were randomly divided into five groups ( $\mathrm{n}=5$ each): sham group, CLP group, Dexmedetomidine group (Dex, $50 \mu \mathrm{g} / \mathrm{kg}$ ) and PTS groups (25 and $50 \mathrm{mg} / \mathrm{kg}$ ). Twenty-hours hours after CLP, PTS was intraperitoneally injected for 14 continuous days. The rats were killed, and blood and lung tissue were collected for pathological analysis and mRNA and protein detection.

Results: Our findings showed that PTS reduced the wet/dry ratio and ameliorated sepsis-induced pulmonary fibrosis (PF), which was associated with improvement of pathological damage in lung tissues. We also observed the inhibitory effect of PTS on apoptosis and release of inflammatory cytokines (i.e., tumor necrosis factor- $\alpha$, interleukin-6, and monocyte chemotactic protein 1). In addition, PTS markedly suppressed the phosphorylation levels of Janus kinase-2 (JAK2) and signal transducer and activator of transcription 3 (STAT3).

Conclusions: Our results indicated that PTS inhibited the PF, apoptosis, and inflammatory response via the JAK2/STAT3 pathway in a sepsis-induced ALI rat model, providing a candidate for drug therapy of sepsis-induced ALI.
\end{abstract}

Keywords: Pterostilbene (PTS); inflammatory response; apoptosis; pulmonary fibrosis (PF); Janus kinase-2/signal transducer and activator of transcription 3; acute lung injury (ALI); cecal ligation and puncture (CLP)

Submitted Jul 10, 2020. Accepted for publication Oct 22, 2020.

doi: $10.21037 /$ atm-20-5814

View this article at: http://dx.doi.org/10.21037/atm-20-5814

\section{Introduction}

Acute lung injury (ALI) complicates many clinical symptoms with high morbidity and mortality (1), and is manifested by pulmonary hypofunction, pulmonary edema, neutrophil infiltration, and alveolar capillary membrane permeability (2).
Sepsis is a complex inflammatory syndrome caused by 6 bacterial infection of the host, which leads to multiple 7 organ dysfunction (3). In their study, Kim et al. reported 8 that sepsis is associated with pulmonary dysfunction (4). ALI is one of the most common complications of sepsis (5), 9

\footnotetext{
$\wedge$ ORCID: 0000-0002-8688-3494.
} 
and the animal model of sepsis-mediated inflammatory lung injury further increases ALI (6). Until recently, the pathophysiological mechanism of sepsis has not been fully elucidated. The inflammatory response triggers the release of a large number of pro-inflammatory cytokines [i.e., tumor necrosis factor- $\alpha$ (TNF- $\alpha$ ), interleukins (ILs), and prostaglandins] in the early stage of sepsis, which plays an important role in the development of sepsis-induced ALI (7). Therefore, it is necessary to seek new treatments for ALI induced by sepsis.

Pterostilbene (PTS) is a natural dimethylated analog of blueberries, and is also found in grapes and peanuts (8). With the progress of bio-utilization, it has been favored by increasing researchers in recent years. Accumulating reports have shown that PTS exhibits multiple biologic activities, including anti-inflammatory, antioxidant, anti-aging, and antiviral activities (9-12). In addition, PTS could mediate the cell cycle, apoptosis, and proliferation to combat various cancers $(13,14)$. Nevertheless, the role of PTS in ALI, such as pulmonary fibrosis (PF), has not been widely researched, and the underlying mechanisms of ALI remain unclear.

$P F$ is caused by abnormal repair [fibroblast hyperproliferation, and massive accumulation of the extracellular matrix (ECM)], and the normal alveolar tissues are subsequently damaged. Keshari et al. reported that sepsis stimulates the process of continuous fibrosis (15). Fibrinolytic imbalance and apoptosis are involved in lung injury and PF, and Bhandary et al. found that apoptotic alveolar epithelial cells irritate the activation and overgrowth of fibroblasts, thereby promoting the occurrence of fibrosis and the progress of PF (16). It has been reported that PTS is protective against hepatic fibrosis and renal fibrosis, but there are few published studies on PF $(17,18)$.

The Janus kinase-2/signal transducer and activator of transcription 3 (JAK2/STAT3) pathway plays a key role in the inflammation-mediated biologic progress. Numerous studies have reported that the JAK/STAT pathway is involved in the development of ALI (19,20).

In the present study, we established a sepsis model induced by cecal ligation and puncture (CLP) to investigate the effects of PTS on ALI. Our study is the first time to explore the protective of PTS on sepsis-induced ALI and its underlying connection with the JAK/STAT pathway. We present the following article in accordance with the ARRIVE reporting checklist (available at http://dx.doi. org/10.21037/atm-20-5814).

\section{Methods}

\section{Main materials and reagents}

PTS $\left(\mathrm{C}_{16} \mathrm{H}_{16} \mathrm{O}_{3}\right.$, molecular weight: $256.3 \mathrm{~g} / \mathrm{mol}$, purity $\geq 99 \%$ ) was purchased from Lifome Technologies LLC (CAS: 537-42-8). Specific pathogen-free male Sprague Dawley rats $(250-300 \mathrm{~g})$ were purchased from the Laboratory Animal Center of Zhejiang University. The antibodies were purchased from Abcam as were as follows: anti-cleaved caspase- 3 antibody (ab49822), anti-caspase-9 antibody (ab184786), anti-B-cell lymphoma-2 (Bcl-2) antibody (ab59348), anti-Bax antibody (ab32503), anti$\alpha$-smooth muscle actin ( $\alpha$-SMA) antibody (ab5694), antifibronectin antibody (ab2413), anti-laminin antibody (ab11575), anti-vimentin antibody (ab24525), anti-collagen I antibody (ab34710), anti-JAK2 antibody (ab108596), antiphospho-JAK2 antibody (ab195055), anti-STAT3 antibody (ab119352), and anti- phospho-STAT3 antibody.

\section{Animal protocol and operation}

The rats were fasted, but had free access to water, 12 hours before the experiment. The protocols were approved by the Ethics Committee of The First Affiliated Hospital of Xi'an Jiaotong University, and all animal surgeries were strictly performed in accordance with Guide for the Care and Use of Laboratory Animals. CLP is the standard model for sepsis, and details of the experimental sepsis model are described elsewhere (21). The rats were randomly divided into five groups $(\mathrm{n}=5$ each): sham group; Dexmedetomidine (22) group (Dex, $50 \mu \mathrm{g} / \mathrm{kg}$ ); CLP group, and PTS (23) groups (25, and $50 \mathrm{mg} / \mathrm{kg}$ ). Twenty-hours hours after CLP, PTS was intraperitoneally injected for 14 continuous days. Sham and CLP group rats were given an equal volume of sterile saline during this period. The rats were then killed, and blood and lung tissue were collected for following studies.

\section{Wet/dry (W/D) ratios}

W/D ratios were measured according to Zhang et al.'s protocol (24). Briefly, immediately after the lungs were removed, the right upper lobe weight (wet weight) was rapidly measured to prevent fluid loss. It was then dried to constant weight in an oven at $60^{\circ} \mathrm{C}$ and the dry weight was measured. The W/D ratio was calculated to assess 
pulmonary edema.

\section{Hematoxylin-eosin (HE) and Masson staining}

Lung tissues were immersed in $10 \%$ formalin for $\geq 48$ hours, and then dehydrated in a concentration gradient of ethanol and embedded in paraffin. Next, $5-\mu m$ lung sections were used for routine $\mathrm{HE}$ and Masson staining. HE staining was used for observing pathological changes in lung tissue, and Masson staining was used for observing pathological changes in lung fibers. Five fields of view were randomized at a magnification of $100 \times$.

\section{Terminal deoxynucleotidyl transferase-mediated digoxigenin-dUTP nick-end labeling (TUNEL) staining}

Lung cells apoptosis was confirmed by TUNEL staining assay according to Gill et al.'s protocol (25). After deparaffinage and hydration, tissue sections were stained with the Beyotime Biotechnology colorimetric TUNEL apoptosis assay kit (C1098), as per the manufacturer's instructions. TUNEL-positive cells were captured using an optical microscope; five fields of view were randomly selected at a magnification of $200 \times$, respectively.

\section{Enzyme-linked immunosorbent assay (ELISA)}

Peripheral blood and lung tissue samples were collected for analyzing inflammatory cytokines. The protein levels of TNF- $\alpha$, IL-6, IL-10, and monocyte chemotactic protein 1 (MCP-1) were measured using an ELISA kit (USCN Life Science), following the manufacturer's recommendations.

\section{Quantitative reverse transcription polymerase chain reaction ( $q R T-P C R)$ analysis}

Total RNA was extracted using the RNAeasy animal RNA isolation kit with spin column (R0036; Beyotime Biotechnology). RNA was then reverse transcribed into cDNA with the BeyoRT III first-strand cDNA synthesis kit with gDNA EZeraser (D7180M; Beyotime Biotechnology), as per the manufacturer's instructions. Finally, DNA was amplified at least three times using the QuantStudio 6 flex real-time PCR system (Cata: 4485697; ThermoFisher Scientific), following the manufacturer's recommendations.

\section{Western blot analysis}

Total protein was isolated from the excised lung tissues by the ProteoPrep total extraction sample kit (Sigma-Aldrich
China LLC). The protein samples were transferred to 148 a polyvinylidene fluoride membrane by sodium dodecyl 149 sulfate polyacrylamide gel electrophoresis. The protein $\quad 150$ expressions of caspase-3, caspase-9, Bcl-2, Bax, survivin, 151 $\alpha$-SMA, fibronectin, laminin, collagen I, JAK2, and STAT3 152 were detected using a standard procedure. The dilution 153 concentration of antibodies used was that recommended 154 by the manufacturer. Protein density was standardized as 155 $\beta$-actin, and phosphorylated protein density as total protein.

\section{Immunobistochemistry}

Lung tissues were immersed in $10 \%$ formalin for $\geq 48$ hours, and then dehydrated in a concentration gradient of ethanol and embedded in paraffin. Next, 5- $\mu$ m lung sections were combined with $3 \%$ hydrogen peroxide for 10 minutes to block endogenous peroxidase. The antigen was then repaired with sodium citrate. Sections were then incubated with a primary antibody and a horseradish peroxidaselabeled corresponding secondary antibody. Finally, the lung sections were stained with 3, 3'-Diaminobenzidine tetrahydrochloride (DAB), and five fields of view were randomly captured at a magnification of $400 \times$ under an optical microscope.

\section{Statistical analysis}

All experiments were conducted in triplicate, and analyses of the results were done using IBM SPSS Statistics version 25.0. Data are presented as the mean \pm standard error of mean. Significant differences between two groups were analyzed using Student's t-test, and in multiple groups they were analyzed using one-way analysis of variance. $\mathrm{P}<0.05$ was defined as statistically significant.

\section{Results}

PTS protects against CLP-induced ALI in rats

We investigated the effect of PTS on CLP-induced lung injury, as shown in Figure 1A. HE staining showed that CLP provoked lung tissue edema, neutrophil infiltration, and alveolar septum thickening compared with the sham group. In addition, the lung injury score and the lung W/D ratio were significantly higher than that of the sham group (Figure 1B,C). After treatment with PTS (25 or $50 \mathrm{mg} / \mathrm{kg}$ ) and Dex $(50 \mu \mathrm{g} / \mathrm{kg})$, we observed that lung tissue injury significantly improved, manifested as a gradual disappearance of tissue edema, decreased neutrophil 
A
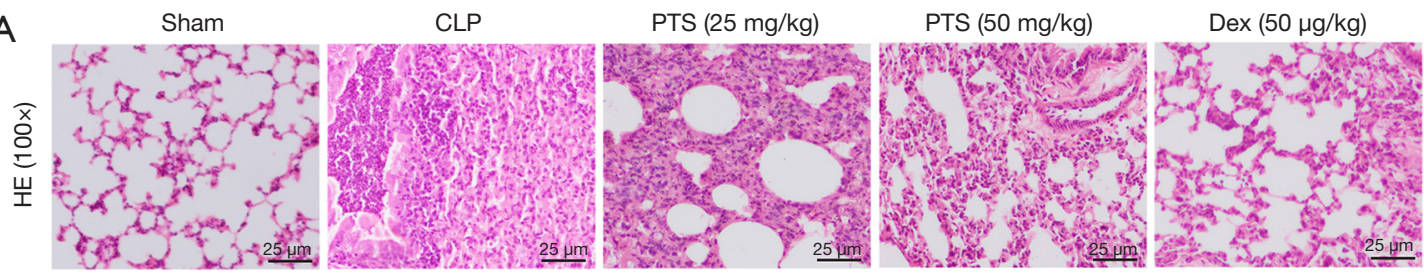

B

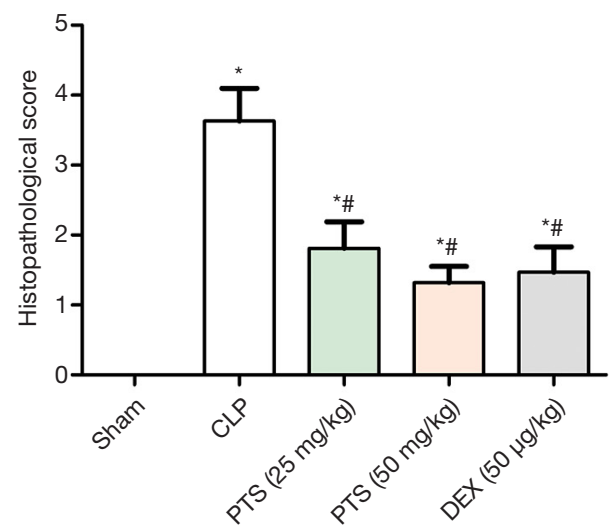

C

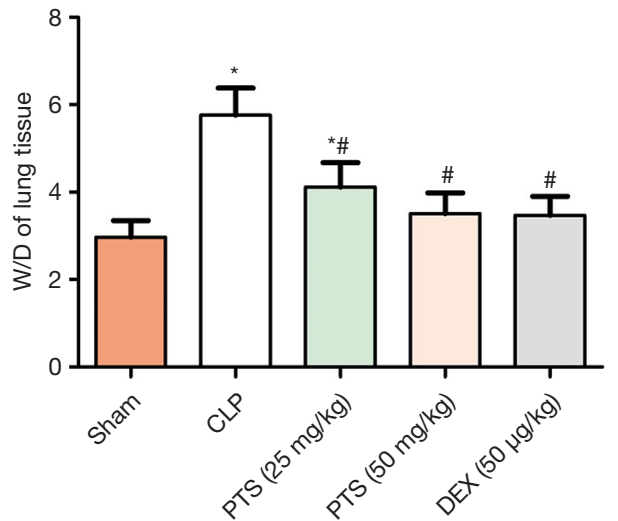

Figure 1 Effect of pterostilbene (PTS) on cecal ligation and puncture (CLP)-induced acute lung injury (ALI). (A) Pathological features in lung tissues were determined using hematoxylin-eosin (HE) staining. Representative images are shown at a magnification of 100x. (B) Lung injury score in each group. (C) Wet/dry (W/D) ratio of CLP-induced lung tissue. Data are shown as the mean \pm standard error of mean $(\mathrm{n}=5) .{ }^{*}, \mathrm{P}<0.05$ versus sham group; ${ }^{\#}, \mathrm{P}<0.05$ versus CLP group. All operations were done in triplicate. IL, interleukin; MCP-1, monocyte chemotactic protein 1 ; TNF- $\alpha$, tumor necrosis factor- $\alpha$.

infiltration, and alveolar septum thinning (Figure 1A). The lung injury score and the lung W/D ratio were also obviously reduced compared with the CLP group (Figure $1 B, C)$.

\section{PTS alleviates CLP-induced inflammatory responses}

Inflammatory cytokines, such as TNF- $\alpha$, IL-6, IL-10, and MCP-1, were also detected in peripheral blood and lung tissues by ELISA assay. In peripheral blood, the TNF- $\alpha$, IL-6, and MCP-1 levels were markedly increased, whereas the IL-10 level was significantly reduced compared with the sham group (Figure $2 A$ ). Conversely, PTS 25 or $50 \mathrm{mg} / \mathrm{kg}$ ) and Dex $(50 \mu \mathrm{g} / \mathrm{kg})$ reduced TNF- $\alpha$, IL- 6 , and MCP1 levels, and increased the IL-10 level. The results for lung tissue were consistent with those of peripheral blood (Figure 2B). In addition, we examined the mRNA levels of IL-6 and IL-10 in lung tissues by qRT-PCR. Similarly, the IL-6 level increased and the IL-10 level decreased in the CLP group, and IL-6 and IL-10 levels were inverted by PTS or Dex treatment (Figure 2C,D).

\section{PTS suppresses CLP-induced cell apoptosis}

To determine the role of PTS on CLP-induced cell apoptosis in lung tissue, we first measured the number of apoptotic cells by TUNEL staining. The positive cell count was remarkably higher than that of the sham group, yet the positive cell count decreased by about $35 \%$ and $40 \%$ after diverse doses of PTS and Dex treatment, respectively (Figure $3 A$ ). We further tested the protein levels of caspase-3, caspase-9, Bcl-2, and Bax by Western blot assay. The expression of cleaved-caspase-3, cleaved-caspase-9, and Bax significantly increased, whereas the expression of Bcl-2 notably decreased in the CLP group (Figure 3B,C). Interestingly, 25 or $50 \mathrm{mg} / \mathrm{kg}$ PTS and $50 \mu \mathrm{g} / \mathrm{kg}$ Dex reversed this change, accompanied by the value of Bcl-2/ Bax increase.

\section{PTS alters the features of CLP-induced PF}

Histological features in the lung were examined by Masson staining, which revealed the presence of PF manifested 
A

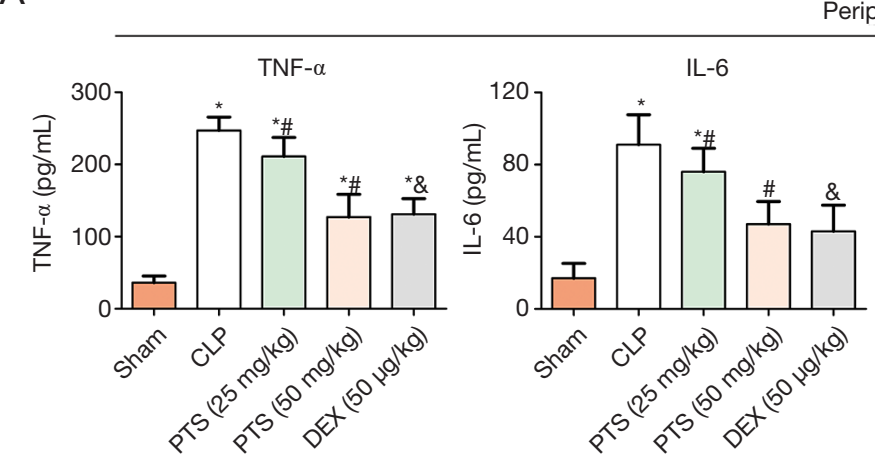

Peripheral blood

B
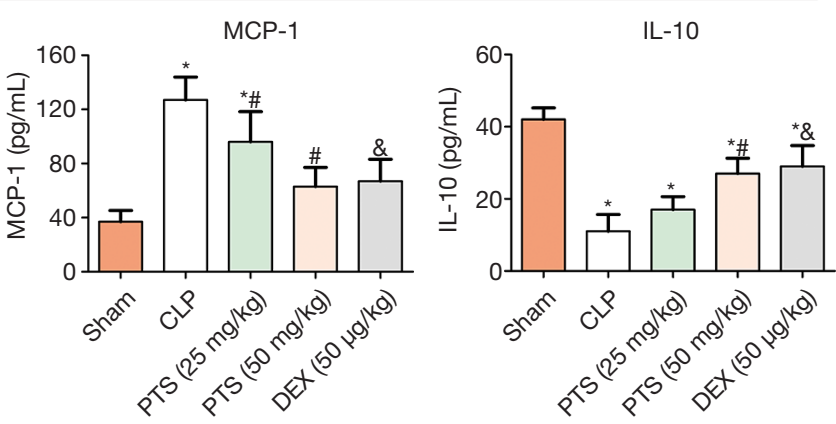

Lung
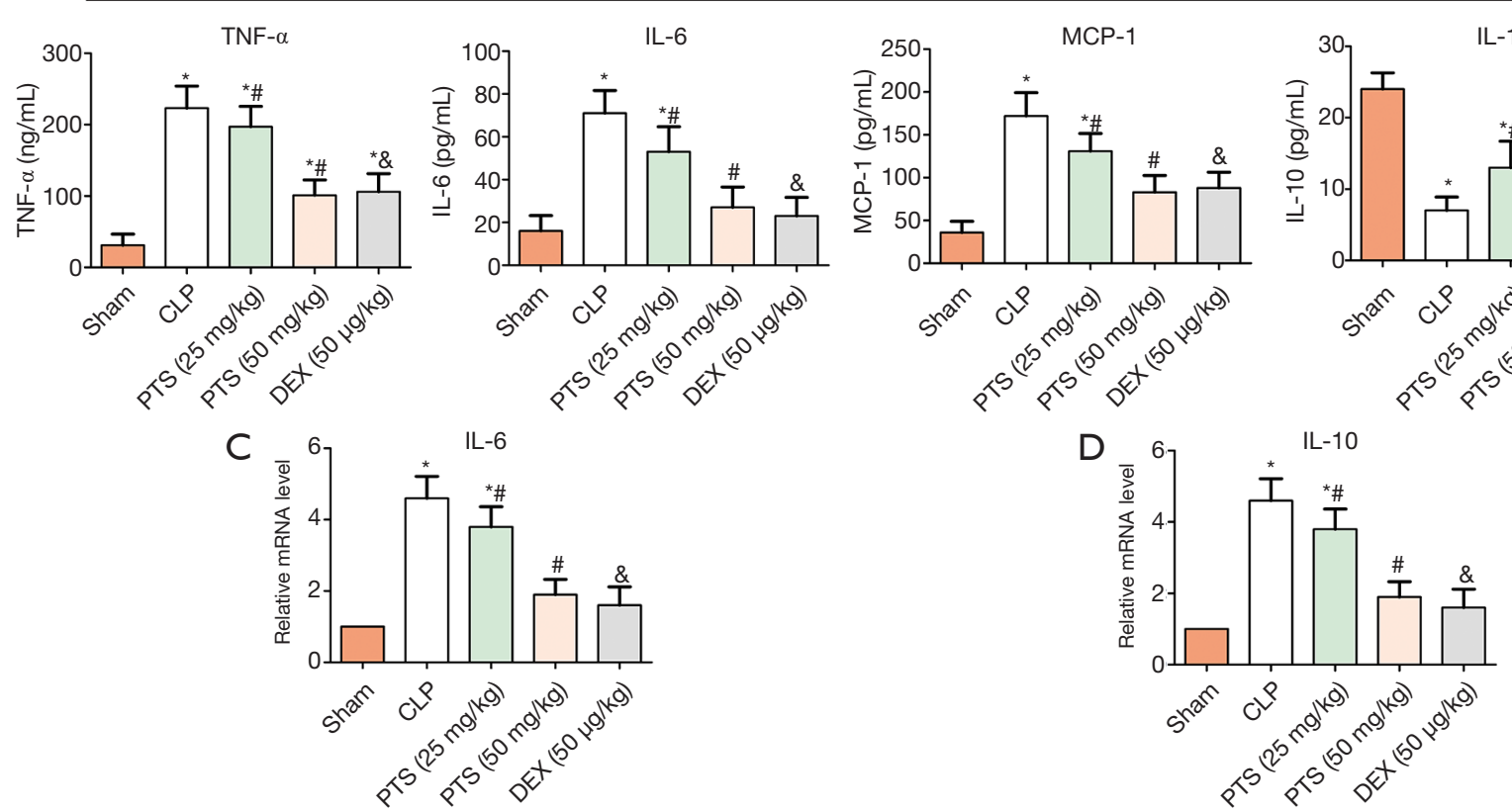

Figure 2 Effect of pterostilbene (PTS) on cecal ligation and puncture (CLP)-induced inflammation. (A,B) Expression of tumor necrosis factor- $\alpha$, interleukin (IL)-6, IL-10, and monocyte chemotactic protein 1 (MCP-1) in peripheral blood and lung tissues were determined using enzyme-linked immunosorbent assay. mRNA levels of IL-6 (C) and IL-10 (D) in lung tissues were determined by quantitative reverse transcription polymerase chain reaction. Data are shown as the mean \pm standard error of mean $(\mathrm{n}=5)$. * $\mathrm{P}<0.05$ versus sham group; ${ }^{\# .8}$, $\mathrm{P}<0.05$ versus CLP group. All operations were done in triplicate. IL, interleukin; MCP-1, monocyte chemotactic protein 1 ; TNF- $\alpha$, tumor necrosis factor- $\alpha$.

as collagen precipitation (Figure $4 A$ ). In contrast, 25 or $50 \mathrm{mg} / \mathrm{kg}$ PTS and $50 \mu \mathrm{g} / \mathrm{kg}$ Dex both mitigated the degree of fibrosis (Figure 4A). We also tested fibrosis markers by Western blot assay, including $\alpha$-SMA, fibronectin, laminin, vimentin, and collagen I. The expression of $\alpha$-SMA, fibronectin, laminin, vimentin and collagen I were notably increased in the CLP group compared with the sham group (Figure 4B,C,D,E,F,G). In PTS and Dex groups, the results revealed that PTS $(25$ or $50 \mathrm{mg} / \mathrm{kg})$ and Dex $(50 \mu \mathrm{g} / \mathrm{kg})$ significantly reduced the protein levels of markers.

\section{PTS inbibits the activation of the CLP-induced FAK2/ STAT3 patbway}

In our study, we found that PTS had an active role on apoptosis, fibrosis, and inflammatory response in ALI. In order to better understand the potential mechanism of PTS in alleviating ALI induced by CLP, we detected the protein expression of JAK2 and STAT3 by Western blot assay. The protein phosphorylation of p-JAK2 and p-STAT3 was higher than that of the sham group, whereas PTS inhibited 
A
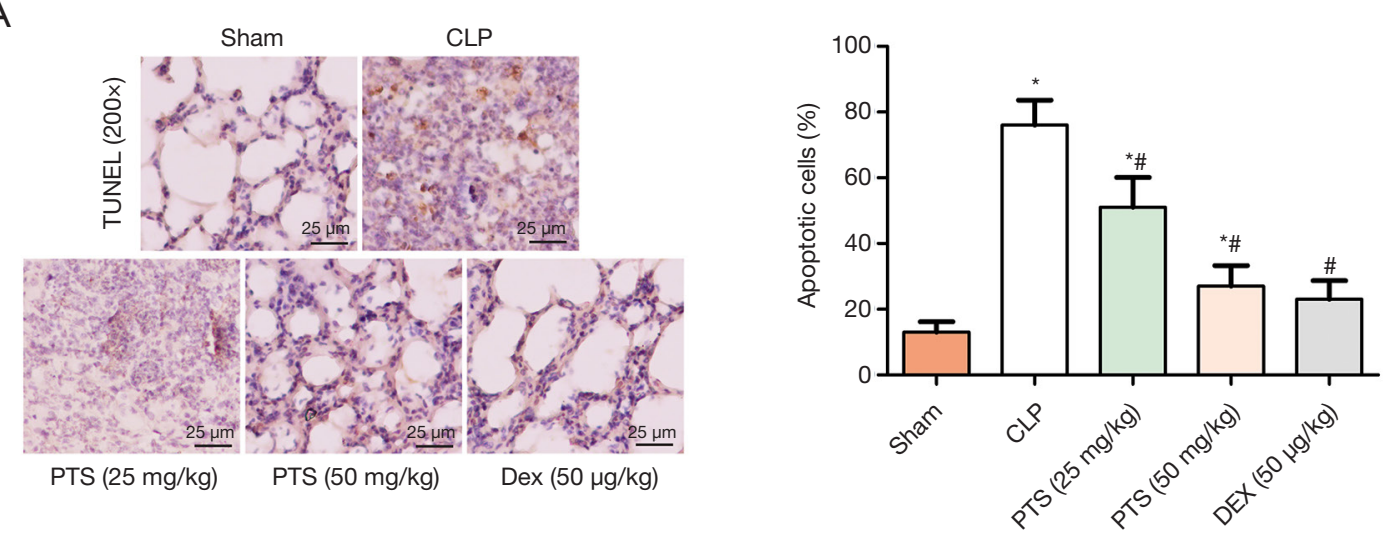

B
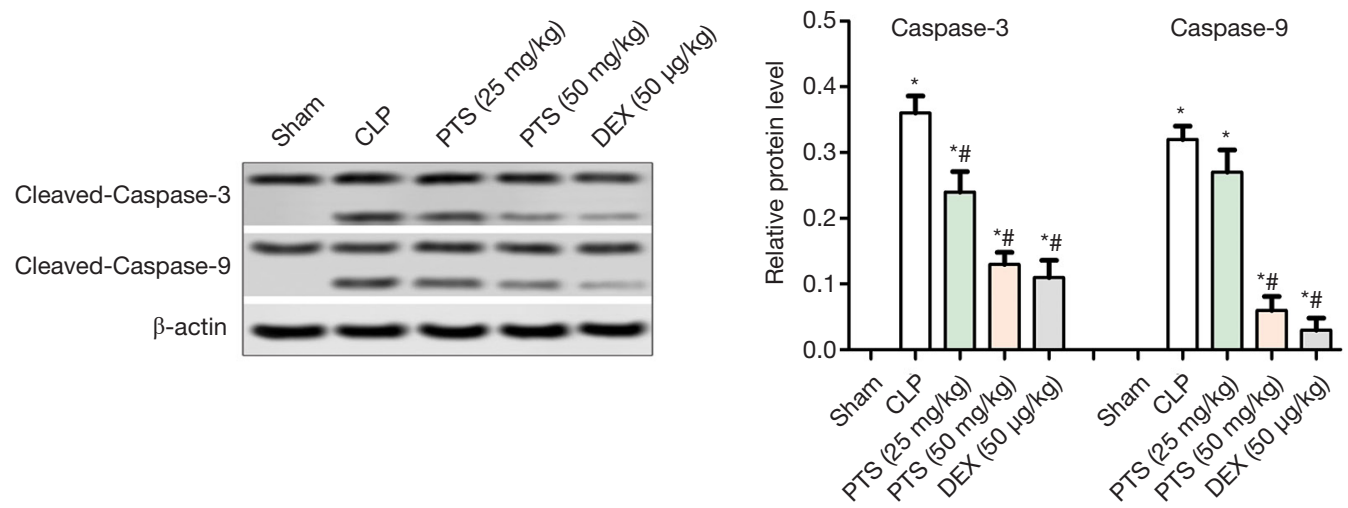

C
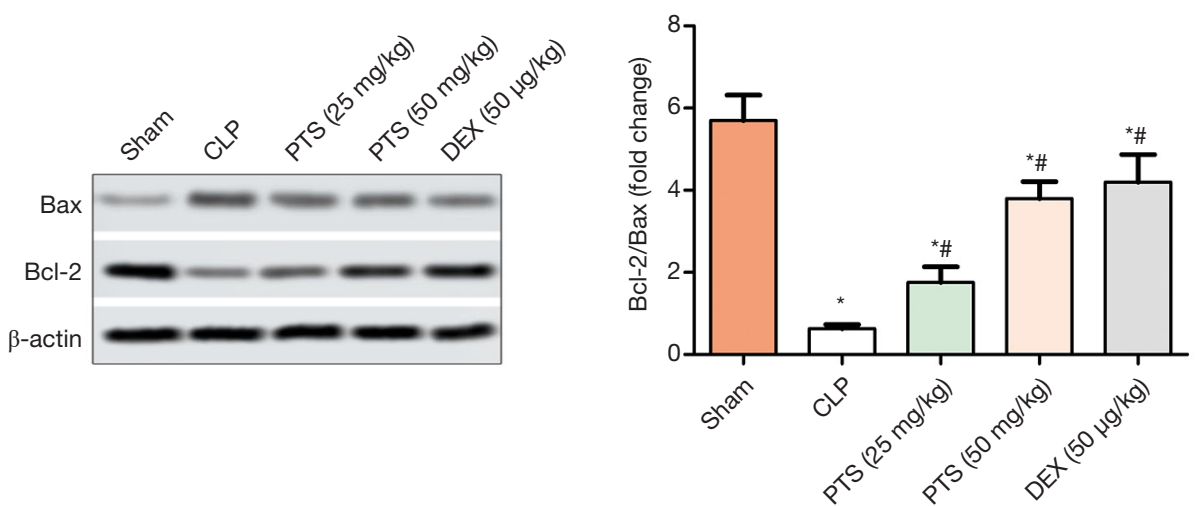

Figure 3 Effect of pterostilbene (PTS) on cecal ligation and puncture (CLP)-induced apoptosis. (A) Cell apoptosis was determined in the lung tissues using terminal deoxynucleotidyl transferase-mediated digoxigenin-dUTP nick-end labeling staining. Nuclei of apoptotic cells were brown. Representative images are at a magnification of 200x. (B,C) Western blot was used to determine the protein levels of cleavedcaspase-3, caspase-9, B-cell lymphoma-2, and Bax. Data are shown as the mean \pm standard error of mean $(\mathrm{n}=5)$. * $\mathrm{P}<0.05$ versus sham group; ", $\mathrm{P}<0.05$ versus CLP group. All operations were done in triplicate. Bcl-2, B-cell lymphoma-2; TUNEL, terminal deoxynucleotidyl transferase-mediated digoxigenin-dUTP nick-end labeling. 
A

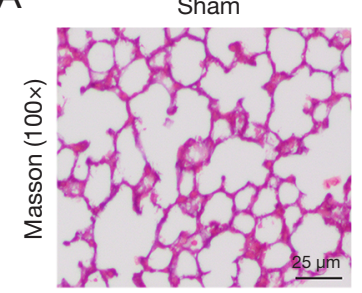

CLP

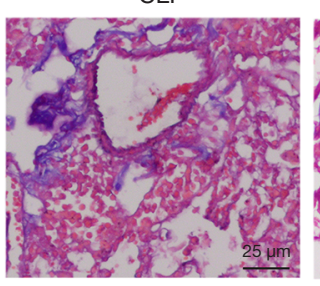

PTS (25 mg/kg)

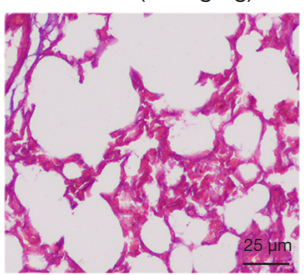

PTS (50 mg/kg)

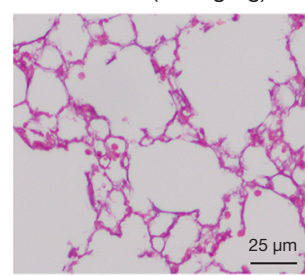

$\operatorname{Dex}(50 \mu \mathrm{g} / \mathrm{kg})$

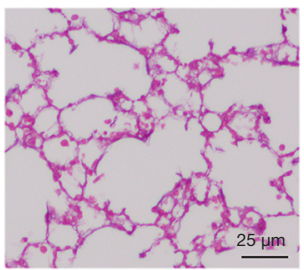

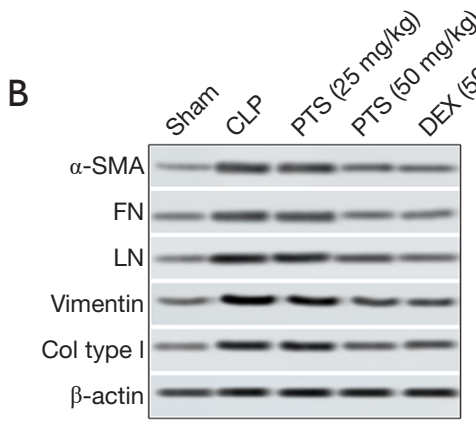

$\mathrm{E}$

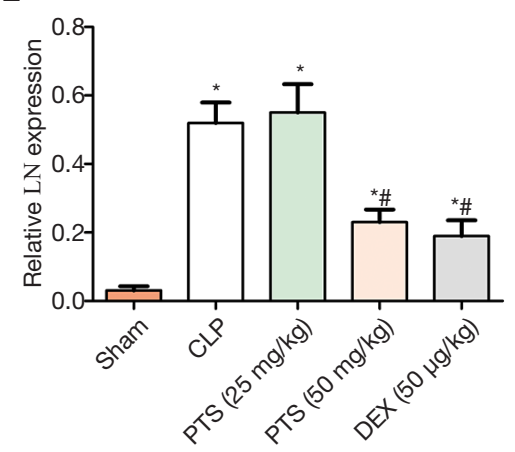

C
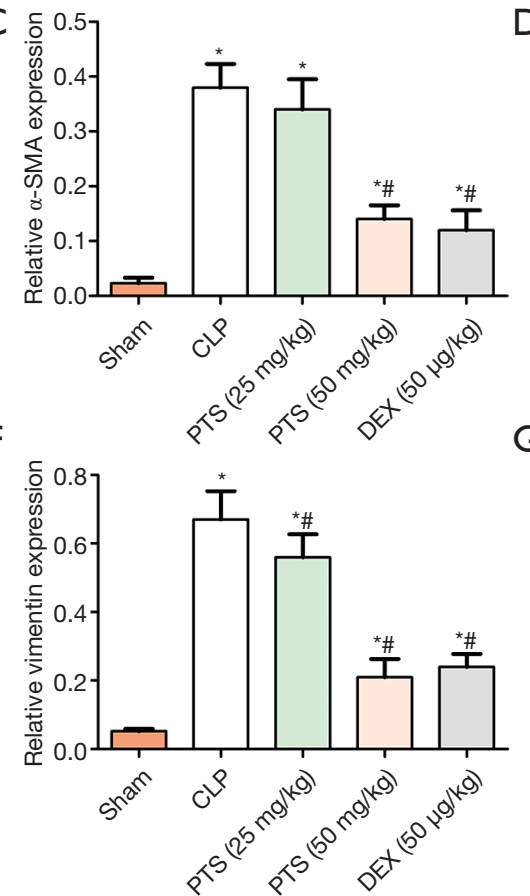

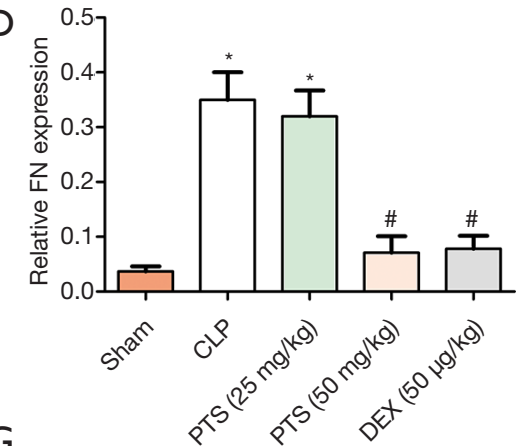

G

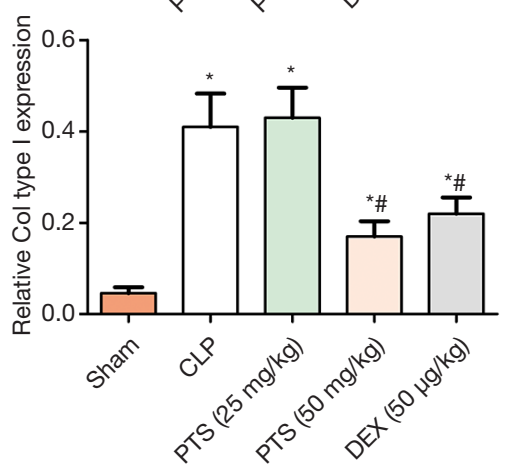

Figure 4 Effect of pterostilbene (PTS) on cecal ligation and puncture (CLP)-induced lung fibrosis. (A) Pathological features in lung fibers were determined by Masson staining. Representative images are at a magnification of 100x. (B,C,D,E,F,G) Protein levels of $\alpha$-smooth muscle actin, fibronectin, laminin, vimentin, and collagen I were determined by Western blot assay. Data are shown as the mean \pm standard error of mean $(\mathrm{n}=5)$. *, $\mathrm{P}<0.05$ versus sham group; ${ }^{*}, \mathrm{P}<0.05$ versus CLP group. All operations were done in triplicate. $\alpha$-SMA, $\alpha$-smooth muscle actin; FN, fibronectin; LN, laminin.

the expression of p-JAK2 and p-STAT3 (Figure 5A). The immunohistochemical results showed that the positive level of p-STAT3 increased in the CLP group. In contrast, compared with the CLP group, PTS reduced the expression of p-STAT3 in a dose-dependent manner (Figure $5 B$ ).

\section{PTS protects against CLP-induced ALI via activating fAK2/STAT3 patbway}

Further, we added JAK inhibitor (AG-490) by intraperitoneal injection, grouped as below: sham group,
CLP group, PTS (50 mg/kg) group, and AG-490 (20 mg/kg) group. As shown in Figure 6A, the phosphorylation levels of JAK2 and STAT3 were obviously lower in the PTS group and AG490 group than that in the CLP group. PTS, or AG490 alone treatment reduced the level of IL-6 (Figure 6B) and increased IL-10 (Figure 6C), compared with CLP group. Masson staining found the similar results in PTS group and AG490 group, that is, collagen precipitation in lung tissue was significantly reduced, compared with CLP group (Figure 6D). In addition, PTS, or AG-490 alone treatment reduced the positive cell count (Figure 6E). 
A

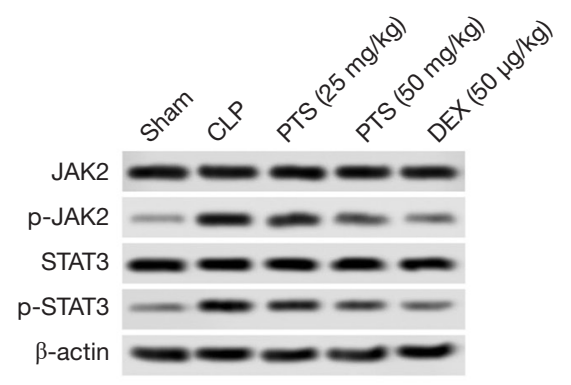

B

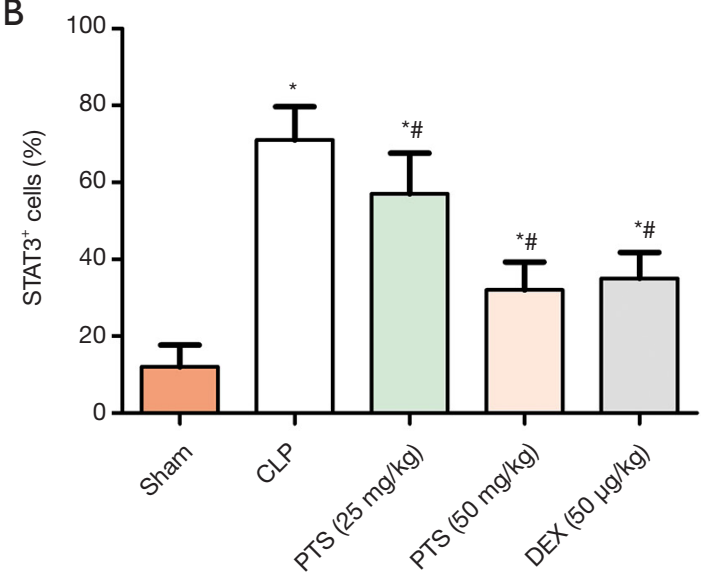

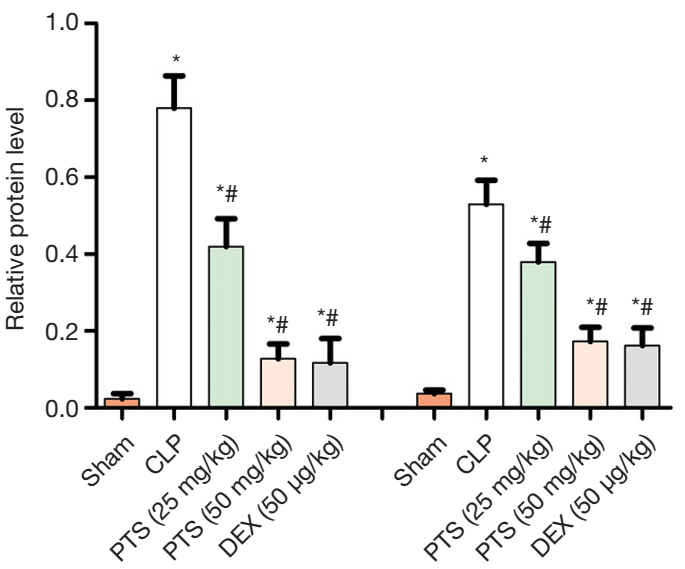

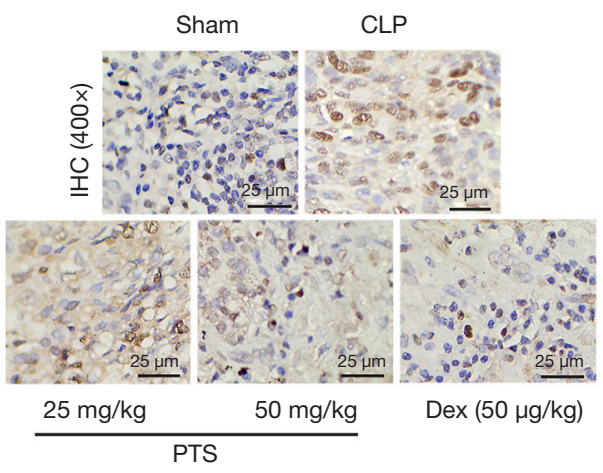

Figure 5 Effect of pterostilbene (PTS) on the phosphorylation of Janus kinase-2 (JAK2) and signal transducer and activator of transcription 3 (STAT3). (A) JAK2, phospho-JAK2, STAT3, and phospho-STAT3 protein levels were determined by Western blot assay. (B) Nuclear positive level of p-STAT3 was determined by immunohistochemistry assay. Representative images are shown at a magnification of 400x. Data are shown as the mean \pm standard error of mean $(n=5)$. *, $P<0.05$ versus sham group; ${ }^{*}, \mathrm{P}<0.05$ versus CLP group. All operations were done in triplicate.

\section{Discussion}

The incidence of sepsis is high and continues to rise, and its pathogenesis is not fully understood. Sepsis causes multiple organ injury, and ALI is a common complication of sepsis. The treatment of the condition is unsatisfactory, and mortality is still high (26). PTS is an active component of blueberries and has various biologic activities; however, the role of opposing lung injury is still poorly understood. In the present study, we investigated the role of PTS against ALI and explored its underlying mechanisms. The results showed that the lung injury score and the W/D ratio notably increased, which proved that the CLP-induced rat model is feasible. After PTS treatment, the lung injury score decreased, whereas that of the W/D weight ratio significantly declined. In previously published papers, pathological features, including lung damage, hypoxia, neutrophil infiltration, and alveolar and interstitial edema, occurred in rats 18-72 hours after CLP induction (2). In the present study, we observed that PTS weakened tissue edema and decreased neutrophil infiltration. We speculated that PTS has a protective effect against sepsis-induced lung injury.

$\mathrm{PF}$ is a serious chronic process that eventually leads to lung injury and respiratory failure. The prominent feature in PF tissues is fibrotic foci, in which active fibroblasts 
A
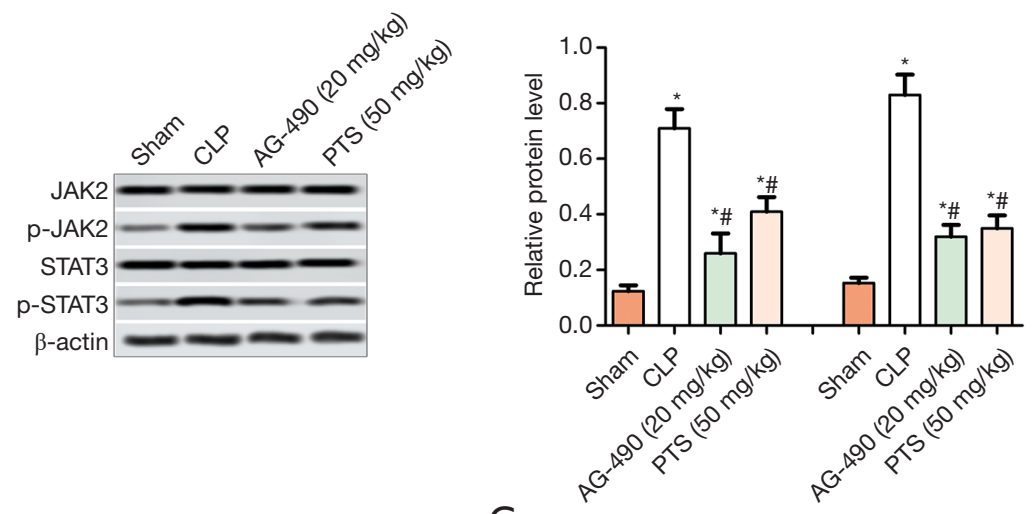

B
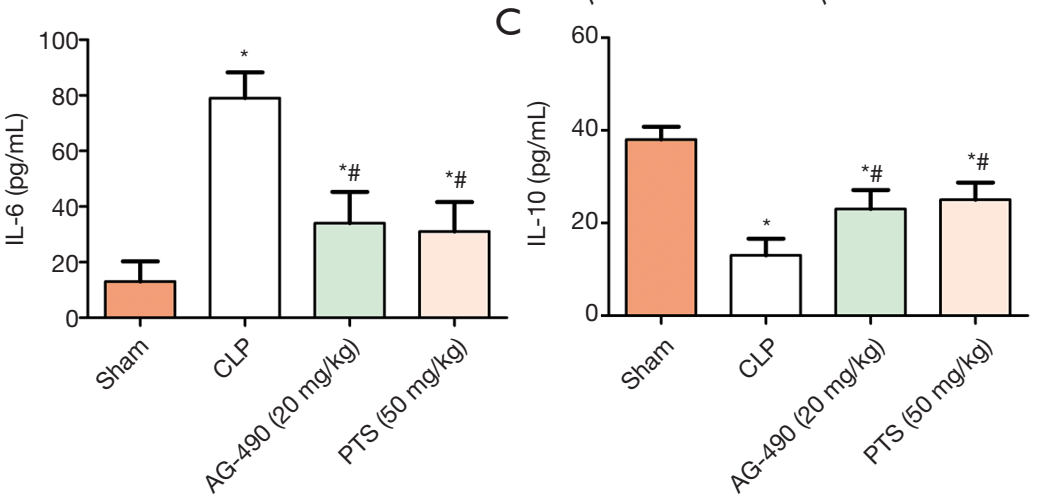

D

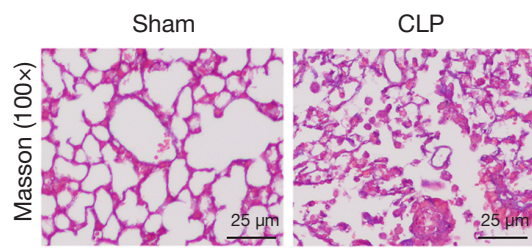

AG-490 $(20 \mathrm{mg} / \mathrm{kg}) \quad$ PTS $(50 \mathrm{mg} / \mathrm{kg})$

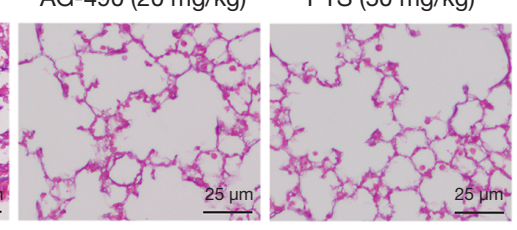

$E$
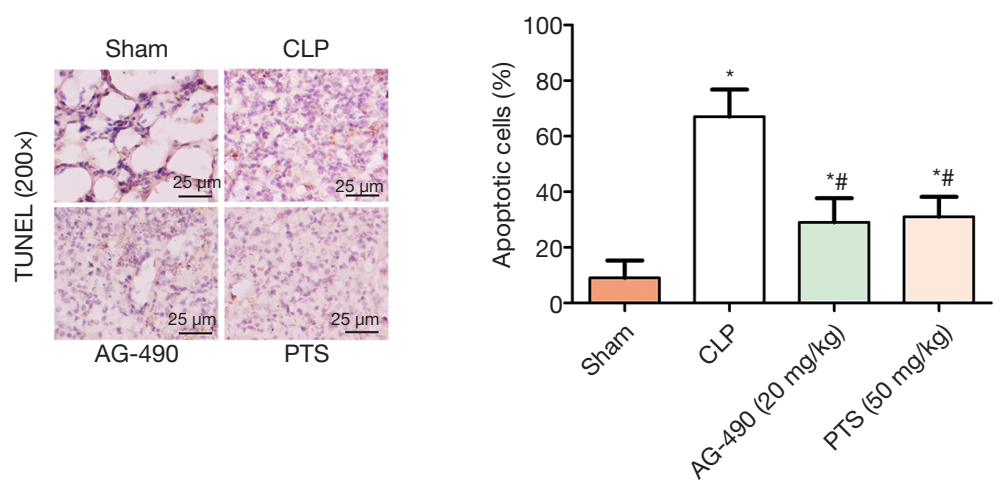

Figure 6 Pterostilbene (PTS) attenuated CLP-induced ALI through inhibiting Janus kinase-2 (JAK2) and signal transducer/activator of transcription 3 (STAT3) Pathway. Post adding JAK inhibitor AG-490 (20 mg/kg). (A) JAK2, phospho-JAK2, STAT3, and phospho-STAT3 protein levels were determined by Western blot assay. (B,C) Expression of interleukin (IL)-6, and IL-10 in lung tissues were determined using enzyme-linked immunosorbent assay. (D) Pathological features in lung fibers were determined by Masson staining. Representative images are at a magnification of 100x. (E) Cell apoptosis was determined in the lung tissues using terminal deoxynucleotidyl transferasemediated digoxigenin-dUTP nick-end labeling staining. Nuclei of apoptotic cells were brown. Representative images are at a magnification of 200x. Data are shown as the mean \pm standard error of mean $(\mathrm{n}=5)$. *, $\mathrm{P}<0.05$ versus sham group; ${ }^{*}, \mathrm{P}<0.05$ versus CLP group. All operations were done in triplicate. 
differentiate into myofibroblasts, resulting in the deposition of collagen and fibronectin in the ECM (27). In a previously published study, it was found that myofibroblasts cause the deposition of excess ECM (i.e., collagen I and collagen III) by increasing $\alpha$-SMA fibronectin and collagen, due to the activation and differentiation of lung fibroblasts, epithelial cell death, and fibrosis remodeling (28). Masson staining revealed the presence of collagen precipitation in CLPinduced lung tissues. The expression of type I collagen and type III collagen increased in patients with idiopathic PF (29). Zhao et al. also reported that the expression of vimentin, a-SMA, Snail, collagen I, and collagen III was upregulated in bleomycin-induced PF (30). Based on this finding, we measured the protein levels of lung fibrous markers ( $\alpha$-SMA, fibronectin, laminin, vimentin, and collagen I). As expected, the results showed that PTS suppressed the expression of $\alpha$-SMA, fibronectin, laminin, vimentin, and collagen I following CLP-induced fibrosis, consistent with previous studies $(29,30)$, indicating that PTS inhibits the ECM accumulation of lung fibroblasts and transforms them into myofibroblasts, thereby demonstrating an anti-PF role.

Lung injury can be caused by endotoxin and other bacterial toxins. Sepsis is an acute inflammation that increases the permeability of pulmonary epithelial cells and rapidly accumulates fluid in the lungs, leading to acute pulmonary edema with interstitial fibrosis. Tashiro et al. found that bleomycin-induced fibrosis releases inflammatory cells. including neutrophils, macrophages. and lymphocytes (31). The activation of neutrophils is intimately connected with inflammatory mediators (TNF- $\alpha$, IL- $1 \beta$, and IL-6) and chemokines (IL-8 and MCP-1), which play an important role in ALI (32). Moreover, macrophages recruit profibrotic cytokines, such as TNF- $\alpha$ or/and IL- 6 , to provide a microenvironment for fibrosis (33); TNF- $\alpha$ and IL-6 reflect the degree of inflammation of the body. TNF- $\alpha$, IL-1 $\beta$, IL- 6 , and nitric oxide are vital pro-inflammatory cytokines, and the interaction between these cytokines, accompanied by ampliative cascades, accelerate the progress of sepsisinduced ALI (34), which alters vascular access permeability, leading to the formation of pulmonary edema (35). In the present study, we found that PTS remarkably suppresses the expression of inflammatory cytokines (TNF- $\alpha$, IL-6, and MCP-1) in CLP-stimulated peripheral blood and lung tissues, suggesting that PTS may play a protective role in the lungs through inhibiting the inflammatory response.

Cell apoptosis in pulmonary tissues is involved in the development and progression of lung injury during sepsis (36). The TUNEL results showed that, compared with the CLP group, the apoptosis rate in the PTS group 345 decreased, indicating that exogenous PTS could effectively 346 inhibit pulmonary epithelial cell apoptosis in septic ALI rats. 347 Bcl-2 and Bax are important apoptotic factors of the Bcl- 348 2 family; Bcl-2 is an anti-apoptotic protein, whereas Bax is 349 a pro-apoptotic protein (37). Caspase-3 is a key regulatory protein in the downstream pathway of apoptosis that triggers apoptosis and ultimately mediates cellular apoptosis (38). The results of the present study showed that the expression of Bax was upregulated and Bcl-2 was downregulated, which stimulated caspase- 3 to induce apoptosis in the lungs. In contrast, PTS reversed the procedure, suggesting that PTS suppresses the apoptosis of pulmonary epithelial cells to protect against lung injury. The JAK/STAT pathway is involved in many biologic processes. Previously published studies have reported that JAKs are related to cell signaling and STAT3 kinases are related to cell growth, differentiation, and apoptosis (39). Severgnini et al. found that STAT may be associated with the development of ALI (40). Han et al. demonstrated that JAK2/STAT3 levels were upregulated in a severe acute pancreatitis ALI rat model (41). The results of the present study showed that JAK2 and STAT3 proteins were mainly not phosphorylated and the expression level were low in sham group. After CLP induction, the JAK/ STAT signaling pathway in the lung is activated, which is manifested by the increased phosphorylation levels of JAK2 and STAT3. PTS significantly inhibited the phosphorylation levels of JAK2 and STAT3, similar to AG490, of which inhibited the up-regulation of p-JAK2 and p-STAT3, while the activation of JAK2/STAT3 signaling pathway in CLPinduced ALI rats model $(41,42)$.

\section{Conclusions}

Based on the findings of the present study, we suggest that PTS (25 or $50 \mathrm{mg} / \mathrm{kg}$ ) effectively ameliorates lung dysfunction in rats with sepsis-induced ALI, but there is no significant difference between the two doses, indicating that this use of better high doses is more suitable for future studies. This protective mechanism may be through the JAK2/STAT3 pathway, and attenuates $\mathrm{PF}$ and inhibits inflammation and apoptosis. Therefore, PTS could be considered a suitable drug for ALI treatment. Further studies may be needed to support the animal findings obtained in the present study.

\section{Acknowledgments}

The authors thank The First People's Hospital of Xi'an 
Jiaotong University Health Science Center for use of the laboratory.

\section{Footnote}

Reporting Checklist: The authors have completed the ARRIVE reporting checklist. Available at http://dx.doi. org/10.21037/atm-20-5814

Data Sharing Statement: Available at http://dx.doi. org/10.21037/atm-20-5814

Conflicts of Interest: Both authors have completed the ICMJE uniform disclosure form (available at http://dx.doi. org/10.21037/atm-20-5814). The authors have no conflicts of interest to declare.

Ethical Statement: The authors are accountable for all aspects of the work in ensuring that questions related to the accuracy or integrity of any part of the work are appropriately investigated and resolved. The protocols were approved by the Ethics Committee of The First Affiliated Hospital of Xi' an Jiaotong University, and all animal surgeries were strictly performed in accordance with Guide for the Care and Use of Laboratory Animals.

Open Access Statement: This is an Open Access article distributed in accordance with the Creative Commons Attribution-NonCommercial-NoDerivs 4.0 International License (CC BY-NC-ND 4.0), which permits the noncommercial replication and distribution of the article with the strict proviso that no changes or edits are made and the original work is properly cited (including links to both the formal publication through the relevant DOI and the license). See: https://creativecommons.org/licenses/by-nc-nd/4.0/.

\section{References}

1. Villar J, Sulemanji D, Kacmarek RM. The acute respiratory distress syndrome: incidence and mortality, has itchanged? Curr Opin Crit Care 2014;20:3-9.

2. Herrero R, Sanchez G, Lorente JA. New insights into the mechanisms of pulmonary edema in acute lung injury. Ann Transl Med 2018;6:32.

3. Shankar-Hari M, Phillips GS, Levy ML, et al. Developing a new definition and assessing new clinical criteria for septic shock: For the third international consensus definitions for sepsis and septic shock (sepsis-3). JAMA
2016;315:775-87.

4. Kim WY, Hong SB. Sepsis and Acute Respiratory Distress Syndrome: Recent Update. Tuberc Respir Dis (Seoul) 2016;79:53-7.

5. Erickson SE, Martin GS, Davis JL, et al. Recent trends in acute lung injury mortality: 1996-2005. Crit Care Med 2009;37:1574-9.

6. Zhao YD, Huang X, Yi F, et al. Endothelial FoxM1 mediates bone marrow progenitor cell-induced vascular repair and resolution of inflammation following inflammatory lung injury. Stem Cells 2014;32:1855-64.

7. Jacobi J. Pathophysiology of sepsis. Am J Health Syst Pharm 2002;59:S3-8.

8. Chang J, Rimando A, Pallas M, et al. Low-dose pterostilbene, but not resveratrol, is a potent neuromodulator in aging and Alzheimer's disease. Neurobiol Aging 2012;33:2062-71.

9. Lv M, Liu K, Fu S, et al. Pterostilbene attenuates the inflammatory reaction induced by ischemia/reperfusion in rat heart. Mol Med Rep 2015;11:724-8.

10. McCormack D, McFadden D. A review of pterostilbene antioxidant activity and disease modification. Oxid Med Cell Longev 2013;2013:575482.

11. Li YR, Li S, Lin CC. Effect of resveratrol and pterostilbene on aging and longevity. Biofactors 2018;44:69-82.

12. Chan CN, Trinité B, Levy DN. Potent inhibition of $\mathrm{HIV}-1$ replication in resting CD4 $\mathrm{T}$ cells by resveratrol and pterostilbene. Antimicrob Agents Chemother 2017;61:e00408-17.

13. Qian YY, Liu ZS, Yan HJ, et al. Pterostilbene inhibits MTA1/HDAC1 complex leading to PTEN acetylation in hepatocellular carcinoma. Biomed Pharmacother 2018;101:852-9.

14. Daniel M, Tollefsbol TO. Pterostilbene down-regulates hTERT at physiological concentrations in breast cancer cells: potentially through the inhibition of cMyc. J Cell Biochem 2018;119:3326-37.

15. Keshari RS, Silasi-Mansat R, Zhu H, et al. Acute lung injury and fibrosis in a baboon model of Escherichia coli sepsis. Am J Respir Cell Mol Biol 2014;50:439-50.

16. Bhandary YP, Shetty SK, Marudamuthu AS, et al. Regulation of lung injury and fibrosis by $\mathrm{p} 53$-mediated changes in urokinase and plasminogen activator inhibitor-1. Am J Pathol 2013;183:131-43.

17. Lee MF, Liu ML, Cheng AC, et al. Pterostilbene inhibits dimethylnitrosamine- induced liver fibrosis in rats. Food Chem 2013;138:802-7. 
18. Pan J, Shi M, Li L, et al. Pterostilbene, a bioactive component of blueberries, alleviates renal fibrosis in a severe mouse model of hyperuricemic nephropathy. Biomed Pharmacother 2019;109:1802-8.

19. Li C, Bo L, Li P, et al. Losartan, a selective antagonist of AT1 receptor, attenuates seawater inhalation induced lung injury via modulating JAK2/STATs and apoptosi in rat. Pulm Pharmacol Ther 2017;45:69-79.

20. Song Z, Zhao X, Gao Y, et al. Recombinant human brain natriuretic peptide ameliorates trauma-induced acute lung injury via inhibiting JAK/STAT signaling pathway in rats. J Trauma Acute Care Surg 2015;78:980-7.

21. Rittirsch D, Huber-Lang MS, Flierl MA, et al. Immunodesign of experimental sepsis by cecal ligation and puncture. Nat Protoc 2009;4:31-6.

22. Liu J, Huang X, Hu S, et al. Dexmedetomidine attenuates lipopolysaccharide induced acute lung injury in rats by inhibition of caveolin-1 downstream signaling. Biomed Pharmacother 2019;118:109314.

23. Yang H, Hua C, Yang X, et al. Pterostilbene prevents LPSinduced early pulmonary fibrosis by suppressing oxidative stress, inflammation and apoptosis in vivo. Food Funct 2020;11:4471-84.

24. Zhang Y, Yu W, Han D, et al. L-lysine ameliorates sepsisinduced acute lung injury in a lipopolysaccharide-induced mouse model. Biomed Pharmacother 2019;118:109307.

25. Gill SE, Rohan M, Mehta S. Role of pulmonary microvascular endothelial cell apoptosis in murine sepsisinduced lung injury in vivo. Respir Res 2015;16:109.

26. Dupuis C, Sonneville R, Adrie C, et al. Impact of transfusion on patients with sepsis admitted in intensive care unit: a systematic review and meta-analysis. Ann Intensive Care 2017;7:5.

27. Murtha LA, Schuliga MJ, Mabotuwana NS, et al. The processes and mechanisms of cardiac and pulmonary fibrosis. Front Physiol 2017;8:777.

28. Phillips RJ, Burdick MD, Hong K, et al. Circulating fibrocytes traffic to the lungs in response to CXCL12 and mediate fibrosis. J Clin Invest 2004;114:438-46.

29. Liu L, Tang L, Xu DS, et al. Effect of radix rehmanniaeon expression of collagen I and III of pulmonary interstitial fibroblast in rat. Chinese Traditional Patent Medicine 2008;30:175-8.

30. Zhao X, Qu G, Song C, et al. Novel formononetin-7-sal ester ameliorates pulmonary fibrosis via MEF2c signaling pathway. Toxicol Appl Pharmacol 2018;356:15-24.

31. Tashiro J, Rubio GA, Limper AH, et al. Exploring animal models that resemble idiopathic pulmonary fibrosis. Front Med (Lausanne) 2017;4:118.
32. Perl M, Lomas-Neira J, Venet F, et al. Pathogenesis of indirect (secondary) acute lung injury. Expert Rev Respir Med 2011;5:115-26.

33. Wynn TA, Barron L. Macrophages: master regulators of inflammation and fibrosis. Semin Liver Dis 2010;30:245-57.

34. Zhao H, Zhao M, Wang Y, et al. Glycyrrhizic Acid Prevents Sepsis- Induced Acute Lung Injury and Mortality in Rats. J Histochem Cytochem 2016;64:125-37.

35. Olman MA, White KE, Ware LB, et al. Microarray analysis indicates that pulmonary edema fluid from patients with acute lung injury mediates inflammation, mitogen gene expression, and fibroblast proliferation through bioactive interleukin-1. Chest 2002;121:69S-70S.

36. Chang CL, Leu S, Sung HC, et al. Impact of apoptotic adipose-derived mesenchymal stem cells on attenuating organ damage and reducing mortality in rat sepsis syndrome induced by cecal puncture and ligation. J Transl Med 2012;10:244.

37. Low IC, Kang J, Pervaiz S. Bcl-2: a prime regulator of mitochondrial redox metabolism in cancer cells. Antioxid Redox Signal 2011;15:2975-87.

38. Mahadevaiah S, Robinson KG, Kharkar PM, et al. Decreasing matrix modulus of PEG hydrogels induces a vascular phenotype in human cord blood stem cells. Biomaterials 2015;62:24-34.

39. Cambi GE, Lucchese G, Djeokeng MM, et al. Impaired JAK2-induced activation of STAT3 in failing human myocytes. Mol BioSyst 2012;8:2351-9.

40. Severgnini M, Takahashi S, Rozo LM, et al. Activation of the STAT pathway in acute lung injury. Am J Physiol Lung Cell Mol Physiol 2004;286:L1282-92.

41. Han X, Wang Y, Chen H, et al. Enhancement of ICAM 1 via the JAK2/STAT3 signaling pathway in a rat model of severe acute pancreatitis associated lung injury. Exp Ther Med 2016;11:788-96.

42. Song Z, Zhao X, Gao Y, et al. Recombinant human brain natriuretic peptide ameliorates trauma-induced acute lung injury via inhibiting JAK/STAT signaling pathway in rats. J Trauma Acute Care Surg 2015;78:980-7.

(English Language Editor: R. Scott)

Cite this article as: Xue $\mathrm{H}, \mathrm{Li} \mathrm{M}$. Protective effect of pterostilbene on sepsis-induced acute lung injury in a rat model via the JAK2/STAT3 pathway. Ann Transl Med 2020;8(21):1452. doi: 10.21037/atm-20-5814 